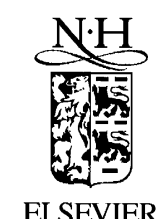

Nuclear Instruments and Methods in Physics Research A 478 (2002) 37-45

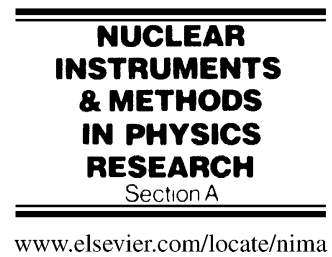

\title{
Overview of silicon detectors
}

\author{
H. Dijkstra \\ PPE Division, CERN, CH-1211 Geneva 23, Switzerland
}

\begin{abstract}
An overview of the ingredients and layout of the most commonly used Si-detectors is given. Their performance is illustrated by the results of some selected experiments. The challenges in the high radiation environment of the LHC, and the changes in signal collection after heavy irradiation are reviewed. Possible future solutions in ultra high radiation environments are briefly discussed. (C) 2002 Elsevier Science B.V. All rights reserved.
\end{abstract}

PACS: 07.77.- $\mathrm{n}$

Keywords: Si sensors; Radiation harness; Charge collection efficiency; Diamond

\section{Introduction}

Semiconductor detectors, and in particular silicon detectors, play an increasingly important role in particle detectors. Heavy particles containing c or b-quarks have lifetimes which allow them to be recognised topologically even in high multiplicity environments. Silicon detectors provide the necessary spatial precision to separate secondary vertices from heavy particle decays from their background. In Section 2, an overview will be given of the various detector techniques employed so far in existing experiments.

Previous detectors did not have to deal with very hostile radiation environments, which are now expected at the new high luminosity colliders. The consequences for the operation of these detectors will be discussed in Section 3, based on the measurements on irradiated diodes and strip detectors. The same radiation environment could lead to very different choices of detector type,

E-mail address: hans.dijkstra@cern.ch (H. Dijkstra). depending on the need for very good precision on the one hand, or large area coverage on the other. The charge collection efficiency of irradiated diodes and segmented detectors will be discussed in Section 4. Future colliders might be able to deliver luminosities which require detectors to be more radiation resistant by an order of magnitude. Ongoing efforts to cope with this environment, like cooling silicon detectors down to cryogenic temperatures, or employing diamond as the detector material, will be described in Section 5 .

\section{Basic ingredients and examples}

Fig. 1 shows the basic principle of the detector. A traversing minimum ionising particle (MIP) liberates on average 90 electron-hole pairs per $\mu \mathrm{m}$, hence for a $300 \mu \mathrm{m}$ thick detector this allows a reasonable signal-to-noise measurement of the induced signal for a radiation length of $0.32 \%$. To achieve good spatial precision, strips are usually implanted at a pitch which matches the 


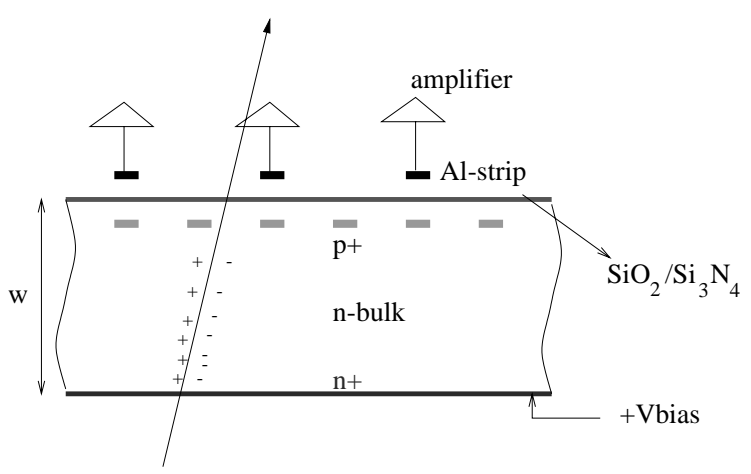

Fig. 1. Cartoon of the basic operation of a n-bulk Si-detector, showing the detector thickness $w$, and $\mathrm{p}+$ implants insulated from the AC-coupled $\mathrm{Al}$ electrodes, which are connected to charge-sensitive amplifiers.

diffusion width of the charge carriers. The diffusion width is proportional to $\sqrt{\frac{\text { mobility }}{\text { drift-time }}}$ and for $w=$ $300 \mu \mathrm{m}$ is typically $10 \mu \mathrm{m}$. This convoluted with the angular spread of the tracks leads to a typical strip pitch of $25 \mu \mathrm{m}$. To reduce the number of electronic channels frequently only every second channel is coupled to an amplifier, since the socalled floating strips have the largest capacitance to their neighbouring strips in the system, hence allowing an accurate position determination of the centre of the gravity of the cluster. For applications which do not require the ultimate precision, pitches of $200 \mu \mathrm{m}$ and larger with a varying number of floating strips in between have been made. Usually, the detectors come with a strip length of a few centimetres up to $10 \mathrm{~cm}$. Pixel detectors with typical pixel sizes of $50 \times 300 \mu \mathrm{m}^{2}$ up to $1 \times 1 \mathrm{~mm}^{2}$ are technologically essential, the same as strip detectors, and are usually used in environments with a high demand on pattern recognition or large occupancies.

Usually, the strips are $\mathrm{p}+$ implants to provide the $\mathrm{p}+\mathrm{n}$-junction in the n-bulk, which are ACcoupled to integrated Al-strips with $\mathrm{SiO}_{2}$, sometimes in combination with $\mathrm{Si}_{3} \mathrm{~N}_{4}$, as an isolation layer. On the ohmic side $\mathrm{n}+$ is implanted to provide the ohmic-contact to bias the detector. The $\mathrm{n}+$ side can also be segmented, usually with orthogonal strips, however, free electrons accumulate at the ohmic side near the $\mathrm{Si}-\mathrm{SiO}_{2}$ surface which causes ohmic short between the $n+$ strips.

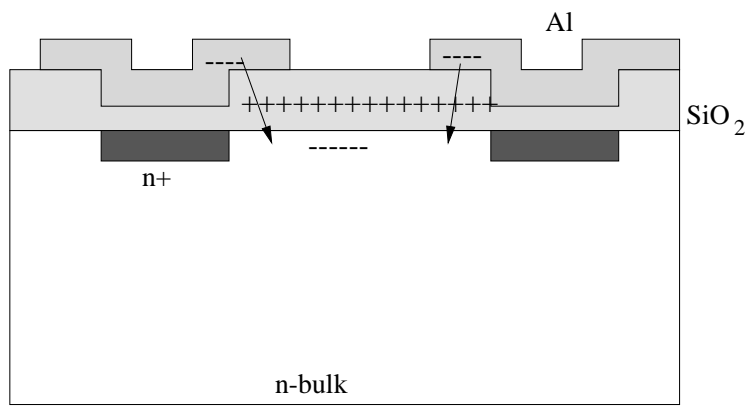

Fig. 2. Extended AC-coupling electrodes at a negative potential compared to the $\mathrm{n}+$ implants interrupt the electron accumulation layer at the $\mathrm{Si}-\mathrm{SiO}_{2}$ surface.

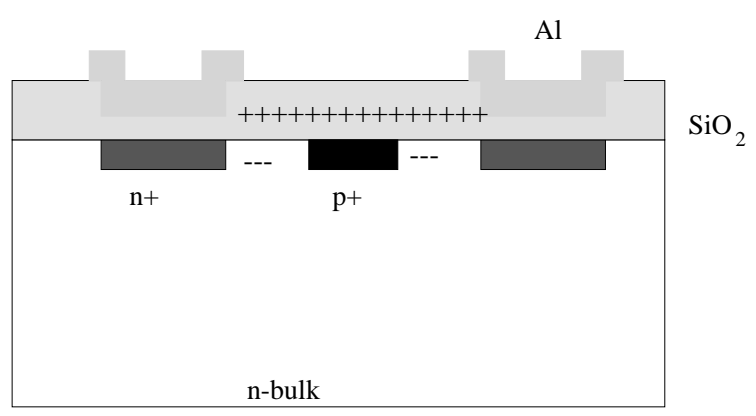

Fig. 3. Schematic of a p-stop grid to achieve an ohmic separation of the $n+$ implants.

Several solutions are used to interrupt this electron accumulation layer. Fig. 2 shows a method which uses extended AC-coupling electrodes, put at a negative potential relative to the $\mathrm{n}+$ strips.

Fig. 3 shows the so-called p-stop design, in which a high dose $\mathrm{p}$-implant is made surrounding the $n+$ strips to interrupt the electron accumulation layer. Originally, only one p-grid was implanted, while an individual $\mathrm{p}$-atoll per $\mathrm{n}+$ strip was found to improve the noise performance of the detectors. Also, combinations of p-stop and field plate separation have been produced to reduce the high field regions after irradiation at the ohmic side of the detector [1]. Another technique neutralises the electrons at the interface by a medium dose p-implant over the whole surface of the detector, which has the added advantage of a lower field near the surface after irradiation. 
The detectors are biased by connecting all strips to a common bias line with a high resistance. Polysilicon resistors are being used, but note that for applications with potentially large currents, i.e. after irradiation, the bias voltage will drop with increased current, while a small value will increase the noise since this is proportional to $\sqrt{\tau / R_{\text {bias }}}$. Low DC resistance combined with high dynamic resistance is obtained with punch-through and Field OXide FET biasing, but it has been shown to be vulnerable to radiation. An alternative to polysilicon is implanted resistors, which simplify the processing [2].

For double sided detectors with orthogonal strips it is usually required to route the signals out of the fiducial volume of the detector, this can be achieved by adding an extra metal layer, insulated from the Al-electrodes by a few $\mu \mathrm{m}$ thick poly-imide or $\mathrm{SiO}_{2}$ layer. Fig. 4 shows a photograph of the corner of the ohmic side of a double sided detector, which uses polysilicon bias resistors, a p-stop grid, and a double metal layer to route the signals to the amplifiers.

Fig. 5 shows the basic layout of the DELPHI SiTracker at LEP [3], which contained practically all

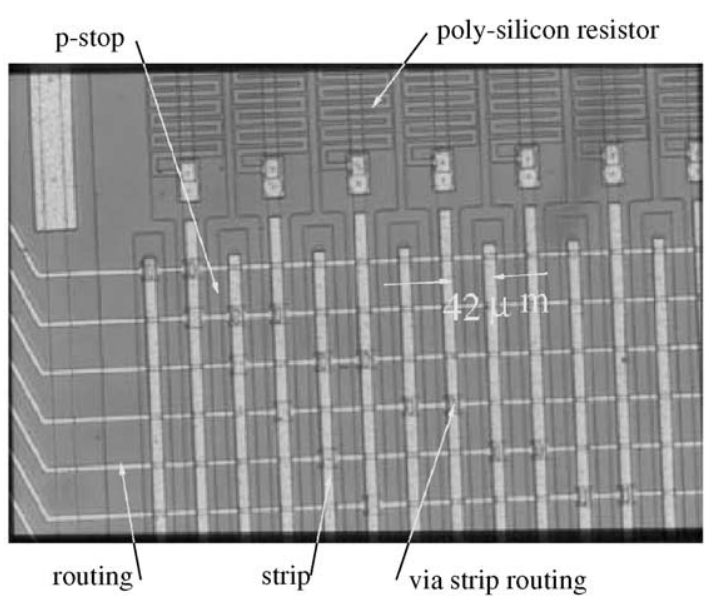

Fig. 4. Photograph of a corner of the ohmic side of a Hamamatsu detector used by DELPHI. A p-stop grid is used to separate the $n+$ implants, which are biased via poly-silicon resistors. The signal is routed to the short edge of the detector with a double metal layer connected to the AC-coupled readout electrodes with vias.

of the techniques discussed above. The detector covers polar angles down to $10^{\circ}$ using a combination of three barrel layers equipped with both

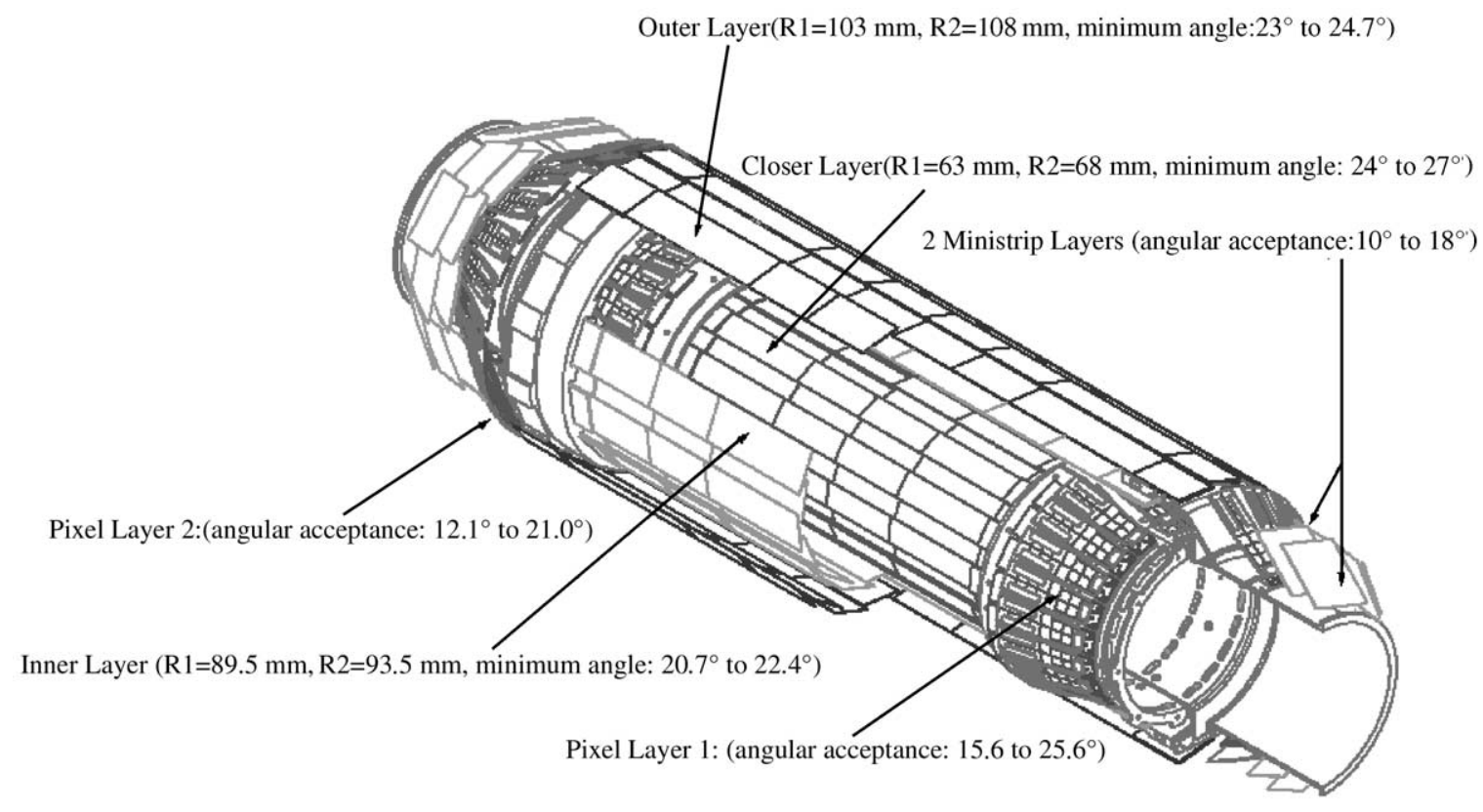

Fig. 5. Layout of the DELPHI Si-Tracker. 
single and double sided strip detectors, and endcaps equipped with a combination of $200 \mu \mathrm{m}$ readout pitch strip detectors and $330 \times 330 \mu \mathrm{m}^{2}$ pixels. A total number of 175,000 electronic channels were used to read out the strips, while $1.2 \times 10^{6}$ pixels were installed for enabling pattern recognition in the limited available space. The total sensitive detector surface was about $1.8 \mathrm{~m}^{2}$.

The SLD vertex detector used Charge Couple Devices (CCD) to reconstruct the $Z^{0}$ decay products at the SLD [4]. The principle of CCD [5] is to capture charge generated in a $20 \mu \mathrm{m}$ thick depletion layer in a potential well just below the surface. By creating a grid of $\mathrm{p}+$ stops and polysilicon gates a three-dimensional device is created. By applying the appropriate sequence of voltages to the gates, the charge can be moved from well to well, till it ends up at the edge of the detector, where it is read out with an amplifier. The small charge (only thin depletion layer) requires the detectors to run cool $(200 \mathrm{~K})$ to reduce the noise from thermally generated electrons. To clock all charge to an amplifier takes a good fraction of an ms, during which the device remains sensitive. The SVX3 of SLD contained $307 \times 10^{6}$ "cubes" of $20 \times 20 \times 20 \mu \mathrm{m}^{3}$ using 96 CCD of $80 \times 16 \mathrm{~mm}^{2}$.

SLD and DELPHI detectors chose very different implementations, driven by the environment at their respective accelerators. A comparison of their performance is given in Table 1. At the SLC the radius of the vacuum chamber was more than a factor of two smaller compared to LEP. Despite this advantage the VXD2 had a similar performance for the multiple scattering term, which was the result of having had to rearrange the detectors at the last moment around a larger beam-pipe than for which the VXD2 had been designed. The asymptotic term is almost three times better than the DELPHI Si-Tracker, but the physics performance is dominated by the multiple scattering term, as is demonstrated in Fig. 6, which shows the b-jet tagging efficiency and purity working point for the measurement of the partial width of the $\mathrm{Z}^{0} \rightarrow \mathrm{b} \overline{\mathrm{b}} .{ }^{1} \quad \mathrm{VXD} 3$ finally exploits the smaller

\footnotetext{
${ }^{1}$ Courtesy of C. Mariotti.
}

Table 1

Comparison of the impact parameter resolution of the DELPHI and SLD vertex detectors in the plane perpendicular to the beam

\begin{tabular}{lll}
\hline Detector & $\mathrm{R}_{\text {beam-pipe }}(\mathrm{mm})$ & $\sigma_{\text {impact }}(\mu \mathrm{m})$ \\
\hline DELPHI & 56 & $\sqrt{28 \oplus 71 /\left(p \times \sin ^{3 / 2} \theta\right)}$ \\
SLD-VXD2 & 25 & $\sqrt{11 \oplus 70 /\left(p \times \sin ^{3 / 2} \theta\right)}$ \\
SLD-VXD3 & 23 & $\sqrt{8 \oplus 33 /\left(p \times \sin ^{3 / 2} \theta\right)}$ \\
\hline
\end{tabular}

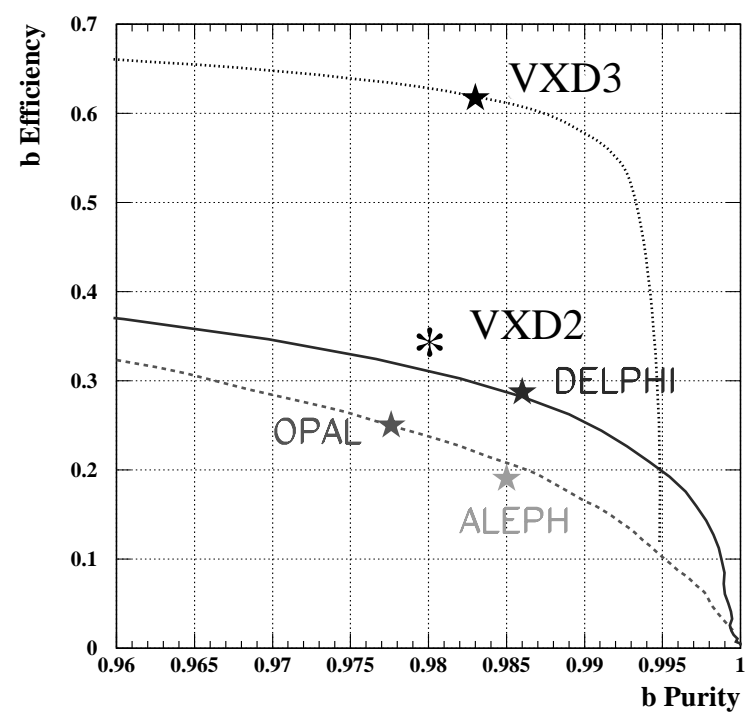

Fig. 6. The b-tagging efficiency and purity working points per hemisphere of ALEPH, DELPHI, OPAL and SLD with $|\cos \theta|<0.7$ for running at the $Z^{0}$.

vacuum chamber, and achieves a twice better efficiency for the same purity.

Heavy ion collision experiments are performed in an environment with large track densities, combined with a low radiation dose. Silicon drift detectors provide a combination of being able to cover large surfaces and provide two-dimensional information with a typical precision of $20-50 \mu \mathrm{m}$. The principle of operation is to implant $\mathrm{p}+$ strips on both side of an n-bulk wafer. A segmented $n+$ diode is implanted at one end of the detector and connected to the amplifiers. Fig. 7 shows a cartoon of the arrangement of the implants [6]. A voltage with a suitable gradient is supplied to the p-strips, 


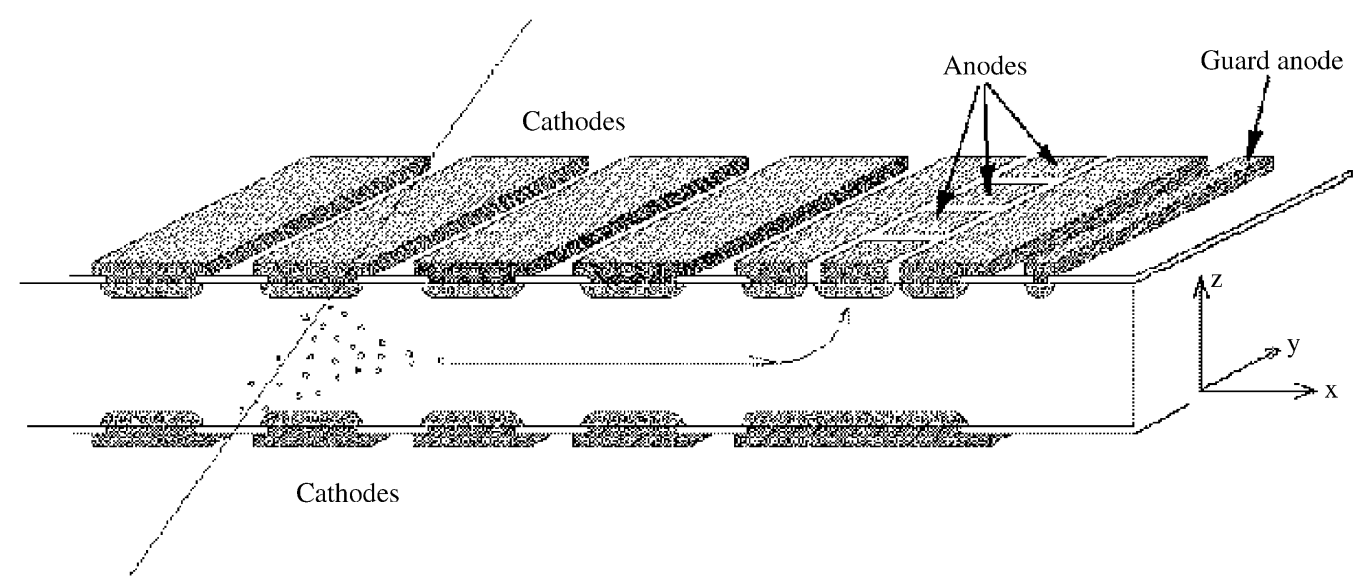

Fig. 7. Schematic of the operation of the Si drift detector. The cathodes are $p+$ implants in $n$-bulk Si. The anodes are $n+$ implants. The $x$-coordinate is given by measuring the drift time, while the $y$-coordinate follows from the segmentation of the anodes.

which creates a potential valley in the mid plane of the detector, sloping towards the $\mathrm{n}+$ implants, where the charge cloud is focused onto the anodes. The $x$-coordinate is measured with the drift time, which is typically a few $\mu \mathrm{s} / \mathrm{cm}$, while the orthogonal coordinate comes from the segmentation of the anodes. Since the charge has to travel a long distance, and one anode collects charge from a large silicon volume the detectors require an excellent material uniformity. To achieve the ultimate resolutions the temperature has to be carefully controlled, and the drift velocity has to be monitored precisely. A two track resolution of 200-600 $\mu \mathrm{m}$ can be achieved, and ALICE plans to install $1.3 \mathrm{~m}^{2}$, segmented in roughly $30 \times 10^{6}$ pixels. $^{2}$ In addition, the drift detectors can provide $\mathrm{d} E / \mathrm{d} x$ measurements, which is an additional advantage for the momentum range of heavy-ion physics.

\section{Radiation}

The LEP machine typically reached a luminosity of $0.3 \times 10^{32} \mathrm{~cm}^{-2} \mathrm{~s}^{-1}$, with the cross-section while running on the $Z^{0}$ pole this gives about $1 \mathrm{e}^{+} \mathrm{e}^{-}$interaction per second. At the high luminosity interaction points at the LHC the luminosity

\footnotetext{
${ }^{2}$ ALICE ITS, CERN/LHCC 99-12, ALICE TDR 4, 1999.
}

is planned to be $10^{34} \mathrm{~cm}^{-2} \mathrm{~s}^{-1}$. This combined with an inelastic non-diffractive cross-section of $55 \mathrm{mb}$ will give $55 \times 10^{7} \mathrm{pp}$ interaction per second. The total particles fluence in the most irradiated parts of the detectors can be as high as $3-4 \times$ $10^{14} \mathrm{~cm}^{-2} \mathrm{yr}^{-1}$. The composition of the particles types and momentum distribution is a strong function of the distance from the interaction point. Typically, at small radii pions are dominating, while for larger radii thermal neutrons cause most of the damage. It is not possible to test the proper functioning of the detectors in an environment equivalent to what is expected at the LHC. Test detectors are irradiated with mostly monochromatic particle beams. To extrapolate from these results to the LHC environment, the expected damage as a function of particle type and momentum is folded into the spectrum expected at the LHC. The radiation damage in silicon is due to the displacements of the lattice atoms and further dynamics. In first order this is due to the first interaction with an atom, and the physical quantity to describe this process is the NonIonising Energy Loss (NIEL). The NIEL for particles can vary many orders of magnitude depending on the particle type and its energy, but it is nearly the same for hadrons with an energy larger than about $1 \mathrm{GeV}$. The NIEL damage functions have been calculated and cross-checked with monochromatic beams over a 
wide range of energies, and take out most of the energy and particles dependence [7]. However, there are large differences seen between neutron and charged hadron induced damage, especially for oxygenated $\mathrm{Si}$, hence one has to be cautious in extrapolating the LHC environment. The standard for particle fluence is to normalise all damage to the equivalent damage caused by $1 \mathrm{MeV}$ neutrons, denoted by $n_{\text {eq. }}$.

Large particle fluences lead to the following macroscopic observables:

- With increasing fluence, the effective space charge in the Si-bulk is decreasing almost linearly with fluence. Since the depletion voltage is proportional to the space charge, this first leads to a decrease in depletion voltage, till the space charge becomes negative (the so-called effective type inversion), and then the voltage increases with fluence. The effective space charge changes with time even after the irradiation has been halted. This change is highly temperature dependent.

- The bulk current increases linearly with the fluence. Unlike the depletion voltage, which is rather difficult to predict for different devices, the current for p-bulk and n-bulk detectors at many different resistivities shows the same dependence on the fluence.

- The carrier lifetime is decreased due to charge trapping, which results in a charge collection inefficiency even at full depletion.

The detector lifetime in a radiation environment is limited by the maximum voltage which can be applied, and by the increase of depletion voltage with fluence. It was found that oxygenated silicon shows less variation and generally lower depletion voltages when irradiated with charged hadrons, however, for neutron dominated fluences no improvement was found [8].

\section{Charge collection efficiency (CCE)}

The charge induced in two parallel electrodes due to a charge $e$ moving in the electric field is $e \times d / w$, where $w$ is the distance between the

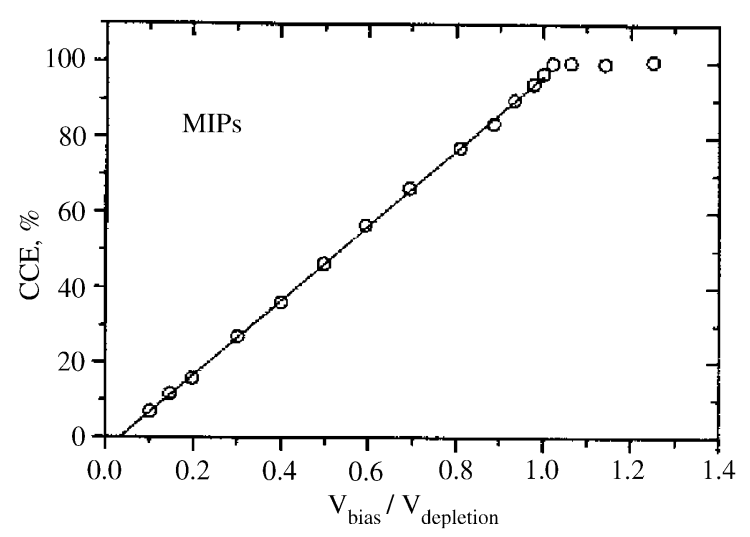

Fig. 8. The CCE for MIPs of a non-irradiated Si-diode as a function of the bias voltage normalised to the depletion voltage.

electrodes and $d$ is the distance over which the charge moves [9]. The free charge carriers in the silicon will only move in a field, hence in the depleted part of the detector. The thickness of the depletion layer is $w \times \sqrt{\frac{V_{\text {bias }}}{V_{\text {depletion }}}}$. Hence for a uniform space charge in the detector this implies that the induced charge is $e \sqrt{\frac{V_{\text {bias }}}{V_{\text {depletion }}}}$. Fig. 8 shows the CCE of a non-irradiated diode as a function of $\sqrt{\frac{V_{\text {bias }}}{V_{\text {depletion }}}}[10]$. The deposited ionisation is proportional to the thickness of the depleted layer; hence, since the CCE is a linear function of $\sqrt{\frac{V_{\text {bias }}}{V_{\text {depletion }}}}$, the apparent separation between the electrodes must be equal to the depleted layer, as it is shown in Fig. 9.

The CCE for diodes irradiated up to fluences of $2.8 \times 10^{14} n_{\text {eq }} \mathrm{cm}^{-2}$ is shown in Fig. 10. For diodes after type inversion the CCE is a linear function of the bias voltage, and Fig. 11 shows that for irradiated diodes the depletion layer grows from the ohmic side. For bias voltages below the depletion voltage, the low-field layer near the junction side of the diode behaves like an insulator for practical peaking times of the amplifiers. Fig. 10 also shows the effect of charge trapping, since even at the full depletion voltage, the CCE is not $100 \%$. Overdepleting the diodes leads to an increase of the drift velocity, hence a shorter charge collection time, and hence a decrease in the fraction of charge carriers which get trapped [11]. The charge division between strips for 


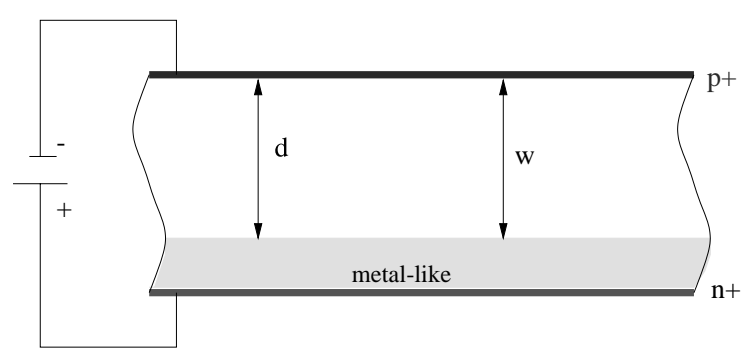

Fig. 9. Schematic of the values of apparent separation between the electrodes $w$, and the thickness of the depletion layer $\mathrm{d}$ for a non-irradiated Si-diode which is not fully depleted.

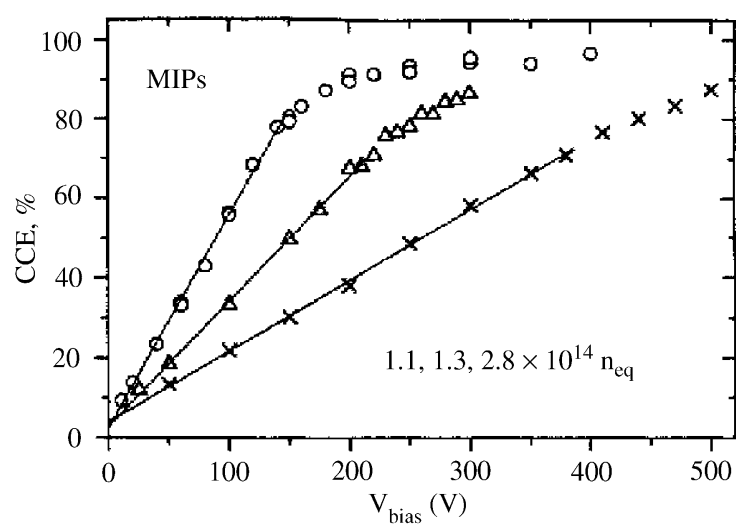

Fig. 10. The CCE for MIPs as a function of the bias voltage for three Si-diodes irradiated to fluences beyond the inversion point.

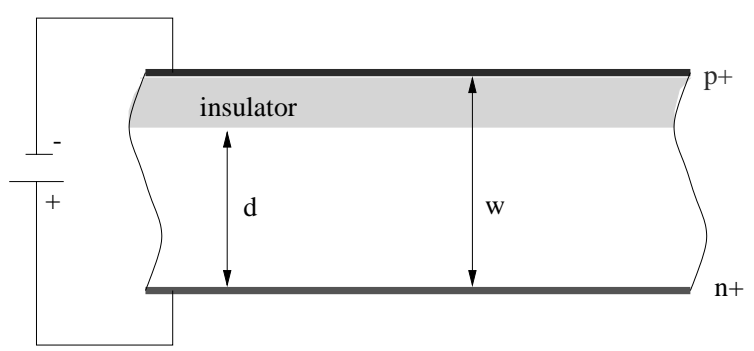

Fig. 11. Schematic of the values of apparent separation between the electrodes $w$, and the thickness of the depletion layer $\mathrm{d}$ for an irradiated $\mathrm{Si}$-diode which is not fully depleted.

non-irradiated devices is solely due to the diffusion of the charge carriers and the incidence angle of the particles. For irradiated detectors the induced charge division is enhanced due to trapping, both on the ohmic and junction sides, while under-

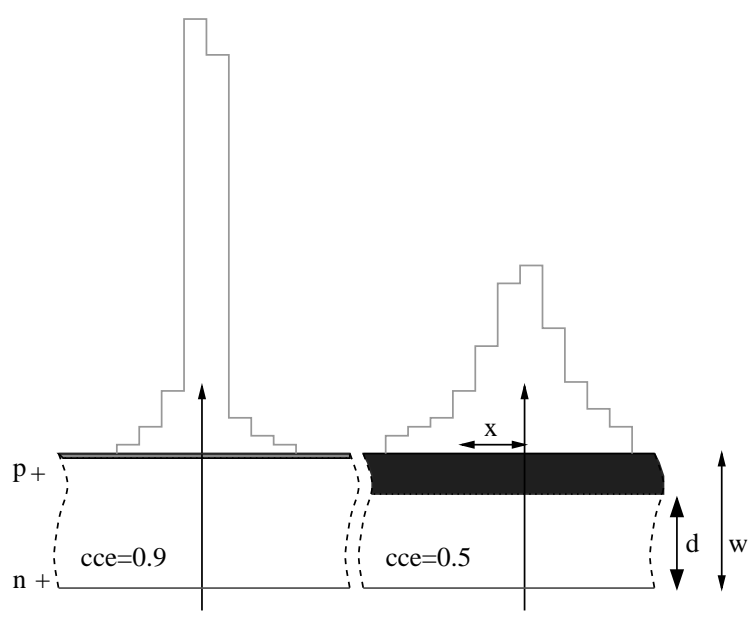

Fig. 12. Schematic of the charge spread on the junction side of a irradiated Si-detector. The total collected charge reduces with lowering the bias voltage below depletion, and the charge is spread over more strips.

depletion causes an extra charge spreading on the junction side [12]. Fig. 12 shows a schematic of the effect of under-depletion on the cluster width on the p-strips [13]. The choice of $p$ or $n$-strip detectors in harsh radiation environments depends not only on the CCE which can be obtained, but also on the strip pitch and consequently the eventual degradation of cluster finding efficiency and resolution as a result of the extra charge sharing on the junction side.

\section{Heavy irradiation}

The present detectors at the LHC have been designed to survive a luminosity of $10^{34} \mathrm{~cm}^{-2} \mathrm{~s}^{-1}$. One of the scenarios to extend the physics reach of the LHC is to increase the luminosity to $10^{35} \mathrm{~cm}^{-2} \mathrm{~s}^{-1}$. As a consequence the detectors need to be able to cope with a magnitude more severe irradiation environment. The depletion voltage increases linearly with the fluence, and the required voltages are much larger than the breakdown voltages reached so far. The number of traps increases with radiation, and the carrier drift velocity saturates above a few $\mathrm{V} / \mu \mathrm{m}$. For fluences larger than a few $10^{14} n_{\mathrm{eq}} \mathrm{cm}^{-2}$ silicon becomes 


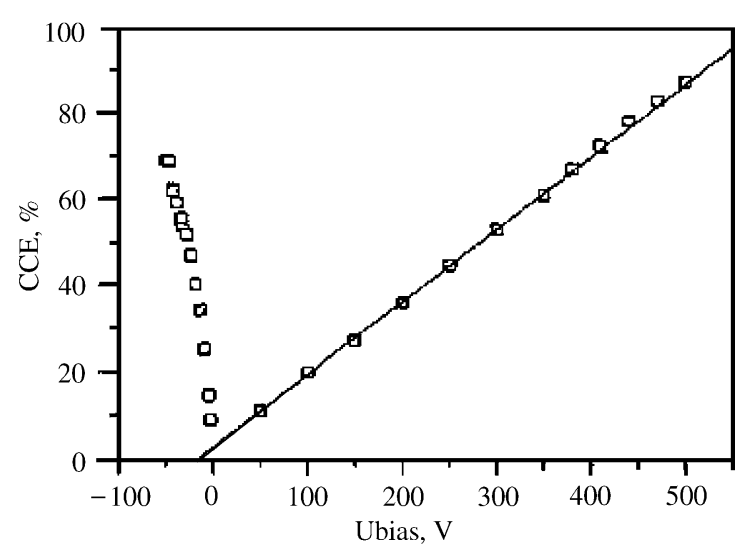

Fig. 13. The CCE of a Si-diode irradiated to $2.8 \times$ $10^{14} n_{\mathrm{eq}} \mathrm{cm}^{-2}$ as a function of both forward (negative bias voltages) and reverse bias operation at $250 \mathrm{~K}$.

ohmic [14], allowing to operate it in forward biased mode. Fig. 13 shows the CCE for a diode irradiated to $2.8 \times 10^{14} n_{\mathrm{eq}} \mathrm{cm}^{-2}$ as a function of both a forward and reverse bias voltage [15]. A $\mathrm{CCE}$ of $70 \%$ is already reached at $50 \mathrm{~V}$ forward bias, while for reserve bias operation of the same diode almost $300 \mathrm{~V}$ is required to reach the same CCE. The carrier lifetime in forward bias mode must have increased an order of magnitude, since at $50 \mathrm{~V}$ the collection time is an order of magnitude larger. A possible explanation is that traps are constantly filled due to the forward current. The consequence on the charge division on segmented devices has not been established, but the CCE for $\alpha$ particles at $50 \mathrm{~V}$ is less than $20 \%$, both for exposing the ohmic and junction sides. This suggests that the field near both surfaces is very low, hence leading to larger induced charge spreads.

The diode of Fig. 13 is operated at $250 \mathrm{~K}$, but in forward biased mode the total current on a surface of $60 \mathrm{~cm} \times 50 \mu \mathrm{m}$ is still $18 \mu \mathrm{A}$, hence to really be able to run at these currents even lower temperatures are necessary to avoid large shot noise. Another way to keep the traps filled is to lower the temperature, since the emission time of deep traps is proportional to $\mathrm{e}^{E_{\text {trap }} / \mathrm{kT}}$. In the first approximation, $\mathrm{CCE} \propto\left(\frac{d}{w}\right)^{2} \times \mathrm{e}^{-t_{\text {collection }} / \tau_{\text {trapping }}}$, and $d^{2} \propto \frac{V_{\text {bias }}}{\left|N_{\text {eff }}\right|}$. At temperatures around $130 \mathrm{~K}$ the trapping and emission imbalance leads to a reduced $\left|N_{\text {eff }}\right|$, which

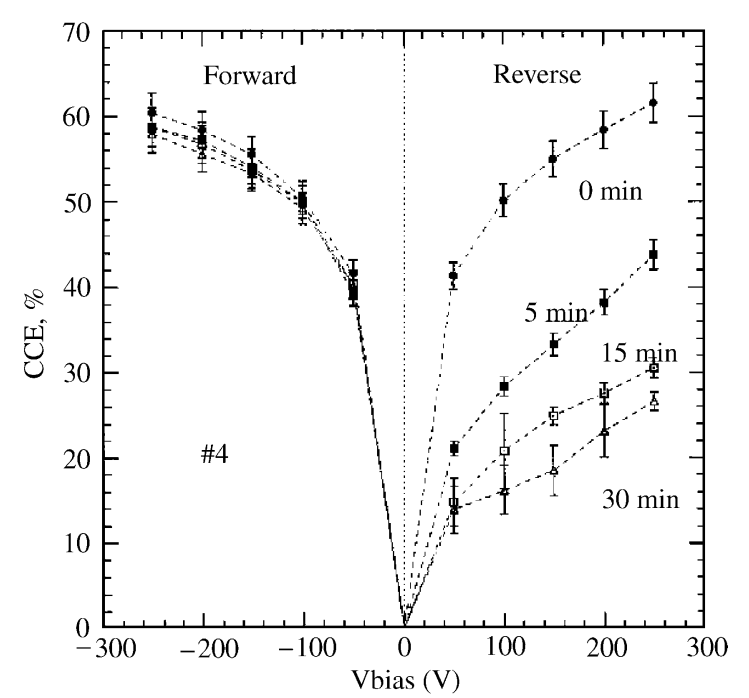

Fig. 14. The CCE of a Si-diode irradiated to $2 \times 10^{15} \mathrm{~cm}^{-2}$ reactor neutrons as a function of both forward (negative bias voltages) and reverse bias operation at $77 \mathrm{~K}$.

has been confirmed using the cluster shapes on the junction side at fluences for which trapping is negligible [13]. However, running at such low temperature leads to such small currents that the trapped emission eventually dominates, leading to very unstable systems as is shown in Fig. 14 [16]. Immediately after applying the bias voltage the reverse bias operation shows a behaviour very similar to the forward bias performance in CCE, but after a few minutes the CCE drops significantly. Inducing some current in the diodes by shining light recovers some of the CCE, but making this a stable system and assuring charge focusing in the case of a segmented device still needs to be established.

Another attempt to survive very harsh radiation environments is chemical vapour deposition (CVD) diamond. Diamond is a good insulator, and typically one applies $1 \mathrm{~V} / \mu \mathrm{m}$ to collect the signal. At these voltages the current is negligible, and practically independent from the fluence. The passage of a minimum ionising particles in diamond liberates 36 electron-hole pairs per $\mu \mathrm{m}$, compared to 89 for Si. In addition the carrier lifetime in CVD diamond is short, leading to incomplete CCE and charge spread on the strips [17]. The CCE of diamond is usually expressed in 


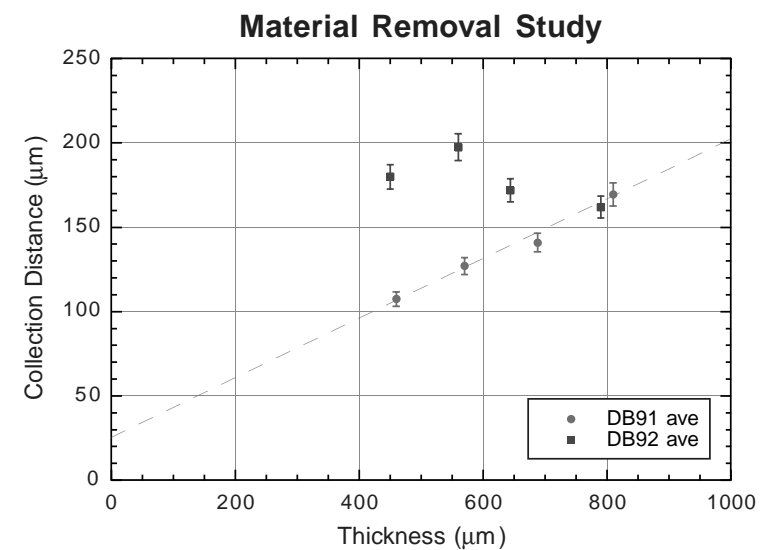

Fig. 15. The collection distance as defined in the text, as a function of the thickness for lapping the CVD diamond down from the nucleation side (full squares) and the growth side (full circles).

terms of the collection depth $\bar{d}=\frac{\text { Signal(e) }}{36 \mathrm{e} / \mu \mathrm{m}}$. The CVD diamonds are grown in thicknesses of typically $800 \mu \mathrm{m}$, and then lapped down. Fig. 15 shows $\bar{d}$ as a function of the thickness of two CVD diamonds, one being lapped down from the nucleation side (full squares), and the other from the growth side (full circles). Lapping down from the nucleation side increases $\bar{d}$, hence the carrier lifetime at the nucleation side is so short that it hardly contributes to the CCE, but decreasing the thickness make the CCE increase. The non-uniformity of the material leads to areas, where the carrier lifetime is even shorter, and causes inefficiencies in cluster finding when running with LHC speed electronics. The charge spreading has been observed in the cluster shapes, and leads to resolutions typically even larger than binary resolution for $50 \mu \mathrm{m}$ strip pitch. A decrease of about $15 \%$ is observed for fluences above $10^{15} 23 \mathrm{GeV}$ protons $\mathrm{cm}^{-2}$. The resolution seems to improve for larger fluences, which is attributed to the more uniform carrier lifetime over the diamond.

\section{Summary}

Silicon detectors have been developed from a few $\mathrm{cm}^{2}$ devices employed 20 years ago to a technique which allows experiments to cover several $100 \mathrm{~m}^{2}$ in relatively harsh radiation environments. Initially, it was envisaged to have btagging at the LHC only for the first few years of low luminosity running, but with the ongoing understanding and improvements of the silicon it is now foreseen to be able to employ the $\mathrm{Si}$ detectors close to the beam throughout the lifetime of the LHC detectors. Apart from standard strip detectors also CCD and Si-drift detectors have found large scale applications depending on the experimental environment. The field is still very active, and new developments like cryogenic operation might still allow the $\mathrm{Si}$ to be used in even more hostile radiation environments. Alternatives, like CVD diamond, have not yet matured enough to challenge silicon, both in price and performance.

\section{References}

[1] P.P. Allport, et al., Nucl. Instr. and Meth. A 450 (2000) 297.

[2] L. Andricek, et al., Nucl. Instr. and Meth. A 436 (1999) 262.

[3] P. Chochula, et al., Nucl. Instr. and Meth. A 412 (1998) 304.

[4] P.N. Burrows, Nucl. Instr. and Meth. A 447 (2000) 194.

[5] C. Damerell, Vertex detectors: state of the art and future prospects, RAL-P-95-008, 1995.

[6] J. Takahashi, et al., Nucl. Instr. and Meth. A 453 (2000) 131.

[7] M. Moll, DESY-THESIS-1999-040, ISSN 1435-8085, 1999.

[8] A. Ruzin, et al., Nucl. Instr. and Meth. A 447 (2000) 116.

[9] G. Cavalleri, et al., Nucl. Instr. and Meth. A 92 (1971) 137.

[10] L. Beattie, et al., Nucl. Instr. and Meth. A 412 (1998) 238.

[11] L. Beattie, et al., Nucl. Instr. and Meth. A 421 (1999) 502.

[12] T.J. Brodbeck, et al., Nucl. Instr. and Meth. A 395 (1997) 29.

[13] K. Borer, et al., Nucl. Instr. and Meth. A 440 (2000) 17.

[14] N. Croitoru, et al., Nucl. Instr. and Meth. B 111 (1996) 297.

[15] A. Chilingarov, et al., Nucl. Instr. and Meth. A 399 (1997) 35.

[16] K. Borer, et al., Nucl. Instr. and Meth. A 440 (2000) 5.

[17] W. Adam, et al., Nucl. Instr. and Meth. A 453 (2000) 141. 